\title{
EFEITO DA CARBONIZAÇÃO SOBRE A ESTRUTURA ANATÔMICA DA MADEIRA DE Alexa grandiflora Ducke
}

\section{EFFECT OF WOOD CARBONIZATION IN THE ANATOMICAL STRUCTURE OF Alexa grandiflora Ducke}

\author{
Marcelo Mendes Braga Júnior ${ }^{1}$; Débora da Silva Souza Santana ${ }^{1}$; Mateus Ferreira Lima ${ }^{1}$; \\ Fernanda Ilkiu Borges de Souza ${ }^{2}$; Luiz Eduardo de Lima Melo ${ }^{3}$.
}

DOI: https://doi.org/10.31692/978-65-991061-7-0.33-41

\begin{abstract}
RESUMO
Este trabalho propõe a caracterização anatômica da madeira e do carvão vegetal da espécie Alexa grandiflora Ducke, visando contribuir para o controle do comércio ilegal de madeira e carvão na Amazônia. Foram selecionados três indivíduos da espécie a partir das amostras de madeira depositadas na Xiloteca IAN (EMBRAPA Amazônia Oriental). Foram preparados corpos de prova para o estudo anatômico do lenho, carbonização e posterior estudo anatômico do carvão. A descrição anatômica da madeira e do carvão seguiu as recomendações preconizadas pela IAWA Committee. O efeito da carbonização sobre a dimensão e quantidade celular da madeira foi avaliada estatisticamente por meio de análise de variância (ANAVA) a 5\% de significância. As características anatômicas qualitativas da madeira foram conservadas mesmo após a carbonização, observou-se que o arranjo do parênquima axial foi a principal característica anatômica para a identificação da madeira e do carvão da espécie. Houveram diferenças estatisticamente significativas para todos os parâmetros anatômicos quantitativos avaliados. De forma geral foi observado redução na dimensão e aumento da quantidade celular após a carbonização da madeira, devido principalmente contrações anisotrópicas do material decorrentes da carbonização. Os resultados apresentam informações uteis para distinção e fiscalização da madeira e do carvão vegetal de A. grandiflora na Amazônia.
\end{abstract}

Palavras-Chave: Fiscalização, Carvão, Amazônia, Conservação da natureza.

\begin{abstract}
\footnotetext{
${ }^{1}$ Engenharia Florestal, Universidade do Estado do Pará, bragajuniorm@ gmail.com

${ }^{2}$ Doutora, EMBRAPA Amazônia Oriental, fernanda.ilkiu@embrapa.br

${ }^{3}$ Doutor, Universidade do Estado do Pará, luizeduardo.limamelo@gmail.com
}

In this work we propose to characterize the anatomical structures of the wood and charcoal of Alexa grandiflora Ducke aiming to contribute to the control of the illegal trade of wood and charcoal in the Amazon. Three individuals of the species were selected from the wood samples deposited in the wood collection IAN (EMBRAPA Eastern Amazon). Test specimens were prepared for the anatomical study of the wood, carbonization and subsequent anatomical study of the charcoal. The anatomical description of wood and coal followed the recommendations recommended by the IAWA Committee. The effect of carbonization on the size and cellular quantity of the wood was evaluated statistically by analysis of variance (ANAVA) at $5 \%$ of significance. The qualitative anatomical characteristics of the wood were conserved even after carbonization, it was observed that the arrangement of the axial parenchyma was the main anatomical characteristic for the identification of wood and charcoal of the species. There were statistically significant differences for all the quantitative anatomical parameters evaluated. In general, a reduction in size and increase in the cellular quantity after carbonization of the wood was observed, mainly due to anisotropic contractions of the material due to carbonization. The results present useful information for the distinction 
and inspection of A. grandiflora wood and charcoal in Amazon.

Keywords: Inspection, Charcoal, Amazon, Nature conservation.

\section{INTRODUÇÃO}

A Amazônia é conhecida por ser detentora da maior extensão de floresta tropical do mundo e também por abrigar uma ampla diversidade de plantas, no entanto, a biodiversidade da floresta amazônica vem acompanhada de intensas taxas de degradação ambiental, aumento do consumo de recursos naturais, crises humanitárias e dificuldades de implementação de atividades ambientais, sociais e econômicas mais sustentáveis, mostrando que os desafios da conservação ambiental são sistêmicos (ICMBIO, 2017). Segundo Hubbel et al. (2008), se o processo de exploração da Amazônia for mantido, há tendência é de extinção de inúmeras espécies, causando, assim, impactos socioeconômicos, culturais e ecológicos para a região.

A vulnerabilidade amazônica aponta a necessidade da disponibilização de literatura sobre as particularidades florestais do bioma, a partir do conhecimento das características das espécies típicas da região é possível realizar estratégias conservacionistas mais eficientes para a flora nativa. Sabendo que a produção ilegal de lenha e carvão vegetal está associada à degradação florestal nesta região (SONTER et al., 2017) os estudos das características anatômicas da madeira e carvão são indispensáveis, pois a partir dessas informações é possível adotar táticas com a finalidade de coibir o comércio ilegal desses produtos.

As dificuldades de fiscalização para a identificação de madeira e carvão vegetal contribuem para o comércio ilegal de carvão vegetal, na Amazônia oriental o comércio de carvão vegetal não certificado coopera com a escravidão, destruição da floresta e desrespeito às comunidades tradicionais (GREENPEACE, 2012). Assim, a disponibilização de informações e capacitação técnica a respeito das características da madeira de espécies florestais amazônicas, assim como de seus derivados, como o carvão vegetal, pode contribuir para a diminuição de impactos sociais e ecológicos.

Portanto, ampliar as informações sobre as características anatômicas e comportamento de madeiras e carvão vegetal oriundo de espécies madeireiras amazônicas é necessário para fomentar a fiscalização, contribuindo assim para o controle do mercado regional e auxiliando a conservação da biodiversidade da região. Pereira et al. (2016) descreveram a anatomia do carvão vegetal de seis clones de Eucalyptus observaram que em maioria as características foram mantidas após o processo de carbonização, fornecendo subsidio para a identificação da madeira que lhe deu origem, entretanto foi observado modificações referente aos parâmetros quantitativos, como o diâmetro dos poros. 
Com base no exposto e considerando a necessidade de estudos que descrevam a estrutura e comportamento do carvão vegetal de espécies da Amazônia, este trabalho teve como objetivo caracterizar as estruturas anatômicas da madeira e do carvão de Alexa grandiflora Ducke, bem como avaliar o efeito da carbonização sob essas características, contribuindo assim para o controle do comércio ilegal de madeira e carvão na Amazônia.

\section{FUNDAMENTAÇÃO TEÓRICA}

A anatomia da madeira está vinculada ao estudo das organizações vegetais que desenvolvem crescimento secundário, ou seja, o conjunto de células, de diferentes tipos que possuem propriedades especificas para desempenhar funções vitais, no tecido lenhoso do corpo do vegetal (BURGUER \& RICHTER, 1991). A anatomia da madeira muitas vezes é utilizada como ferramenta para identificar madeiras contribuindo para fiscalização.

A antracologia é a ciência que estuda resquícios de madeira carbonizada provenientes de solos ou de sítios arqueológicos. A identificação da espécie a qual o carvão é oriundo é realizada a partir da anatomia da madeira, visto que após o processo de carbonização a preservação das características anatômicas muitas vezes é mantida (SCHEEL et al., 1996).

Uma das vertentes do estudo antracológico é direcionada ao setor florestal, fundamentada na caracterização visando à identificação de carvão vegetal, contribuindo para a fiscalização e controle do comércio, o conhecimento das características e comportamento do lenho e carvão vegetal oriundo de espécies florestais colabora para a conservação dos biomas brasileiros (GONÇALVES et al., 2014).

\section{METODOLOGIA}

Nesse trabalho foram utilizados três indivíduos de Alexa grandiflora Ducke, registrado na Xiloteca do Instuto Agronômico do Norte (IAN) da Embrapa Amazônia Oriental e com respectivo material botânico fértil tombado no herbário da mesma instituição. Na Tabela 1 estão listados os indivíduos examinados de acordo com o número da coleção de madeira (xiloteca) e herbário.

Tabela 1: Relação das madeiras analisadas de acordo com o número da coleção de madeira (xiloteca) e herbário. Fonte: Própria

\begin{tabular}{ccc}
\hline Família/Nome científico & Xiloteca, n. & Herbário, n. \\
\hline $\begin{array}{c}\text { Fabaceae } \\
\text { Alexa grandiflora } \text { Ducke } \\
\text { Fabaceae }\end{array}$ & 7789 & 182782 \\
$\begin{array}{c}\text { Alexa grandiflora } \text { Ducke } \\
\text { Fabaceae }\end{array}$ & 7790 & 182801 \\
\hline
\end{tabular}


Alexa grandiflora Ducke

A partir das amostras foram confeccionados corpos de provas com dimensão de 1,5x $1,5 \times 3 \mathrm{~cm}$ (seções tangencial, radial e longitudinal, respectivamente), destes foram confeccionadas lâminas histológicas utilizadas. As amostras de madeira (dimensão: 1,5x $1,5 \times 3 \mathrm{~cm}$ ) foram embrulhadas em papel alumínio, submetidas a um processo de carbonização em forno de mufla com controle automático de temperatura por $\sim 5 \mathrm{~h}$, usando uma taxa de aquecimento de $1.66 \mathrm{C} \min -1$ e taxa de aquecimento de $1,66{ }^{\circ} \mathrm{C} / \mathrm{min}$; mantidas à temperatura final durante $2 \mathrm{~h}$ (MUÑIZ et al., 2012).

As descrições (madeira e carvão) seguiram os procedimentos, e terminologia recomendados pela Associação Internacional de Anatomistas da Madeira (IAWA Committee 1989). As características anatômicas quantitativas analisadas foram: i) diâmetro tangencial dos vasos $(\mu \mathrm{m})$; ii) frequência de vasos por $\mathrm{mm}^{2}$; iii) frequência de raios por mm linear; iv) altura dos raios $(\mu \mathrm{m})$ e v) largura dos raios $(\mu \mathrm{m})$, foram realizadas 30 mensurações para cada parâmetro anatômico avaliado. Foram obtidas imagens da madeira em microscópio de luz transmitida e do carvão em microscópio eletrônico de varredura (MEV) e realizadas medições dos parâmetros anatômicos a partir do software de análise de imagem Image-Pro Express 6.0.

Os dados quantitativos foram submetidos à estatística descritiva para caracterização anatômica do lenho e do carvão. Para verificar o efeito da carbonização $\left(450^{\circ} \mathrm{C}\right.$ e taxa de aquecimento de $1,66{ }^{\circ} \mathrm{C} / \mathrm{min}$ ) sobre a dimensão e quantidade das células da madeira os resultados foram submetidos à análise de variância (ANAVA) a 5\% de significância. Como o estudo envolve apenas um fator (espécie) e este possui apenas 1 grau de liberdade (dois níveis - madeira e carvão), o teste $\mathrm{F}$ é equivalente a qualquer tipo de teste de média, ou seja, o resultado apresentado pelo Teste $\mathrm{F}$ da análise de variância a 5\% de significância é suficiente para testar as hipóteses.

\section{RESULTADOS E DISCUSSÃO}

Observou-se que as características anatômicas qualitativas observadas na madeira foram preservadas mesmo após a carbonização. Assim a caracterização anatômica da madeira se aplica também para o carvão (Figura 1). Anéis de crescimento: distintos, demarcados por finas linhas de parênquima marginal. Parênquima axial: Paratraqueal. Paratraqueal aliforme losangular; ou confluente em trechos curtos oblíquos; ou confluente em trechos longos tendendo a formar faixas. Raios: Finos (menor que 100 $\mu$ m de largura). Baixos (menor do que $1 \mathrm{~mm}$ de altura). Pouco frequentes. Estruturas estratificadas: ausentes. Vasos: solitários e 
múltiplos; arranjo difuso; grandes; pouquíssimo abundantes; parcialmente obstruídos por óleo resina; placas de perfuração simples. Estrutura secretora: ausente. Inclusões minerais: cristais prismáticos presentes em câmaras no parênquima axial.

Figura 1: Micrografia luz transmitida da madeira (a, b, c) e eletromicrografia do carvão vegetal (d, e, f) da espécie Alexa grandiflora. Barra de escala: $200 \mu \mathrm{m}(\mathrm{a}, \mathrm{b}, \mathrm{c}, \mathrm{d}), 100 \mu \mathrm{m}(\mathrm{d}, \mathrm{f})$
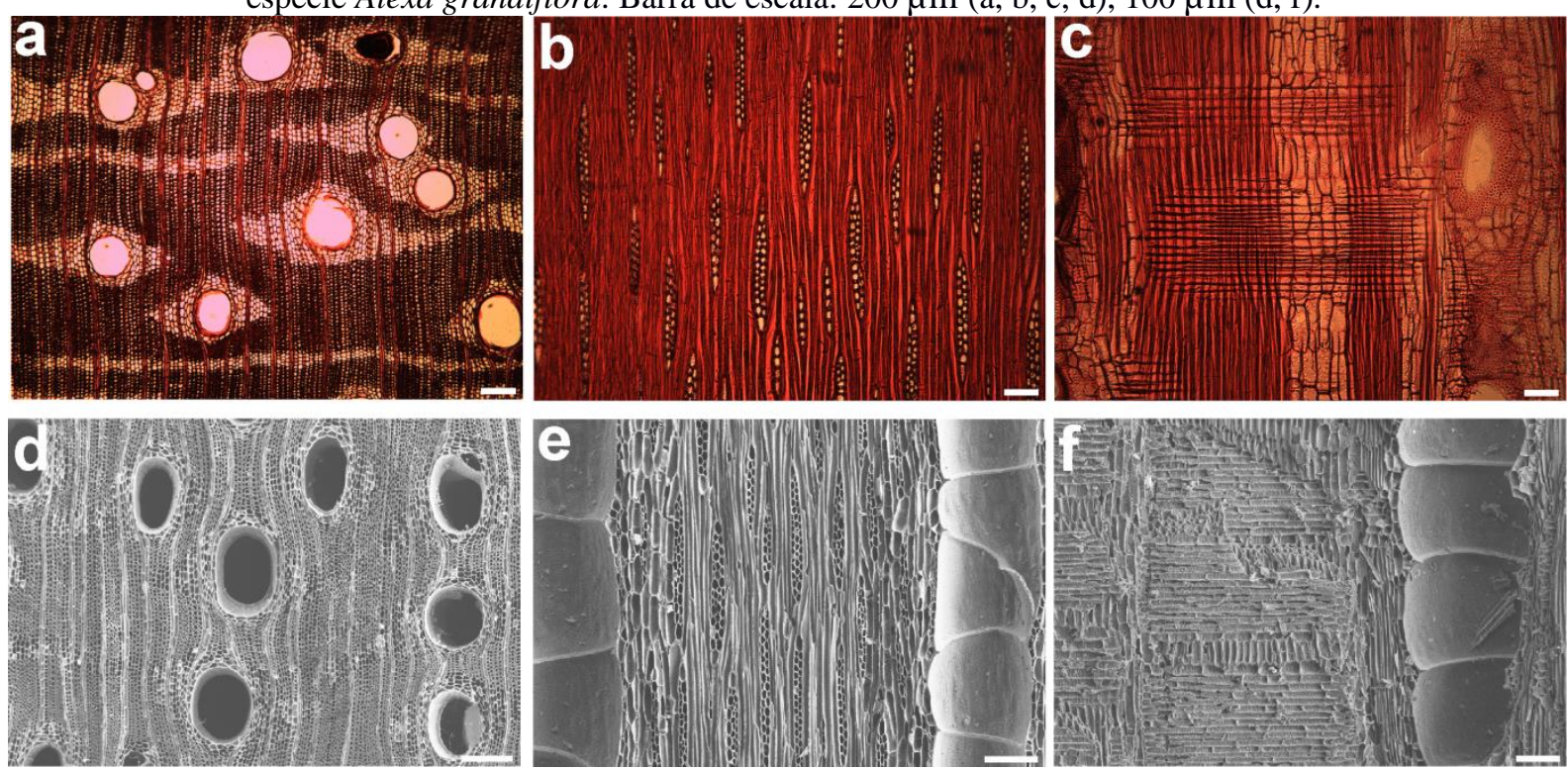

As características anatômicas observadas na madeira e também no carvão, são semelhantes às observações feitas por Fedalto et al. (1989) com exceção da presença não observada pelos autores de anéis de crescimento distintos para espécie, para os indivíduos de A. grandiflora deste estudo os anéis crê crescimento foram distintos e bem demarcados por um parênquima axial confluente em trechos longos tendendo a formar faixas marginal ou simulando faixas marginais, como também foi observado por Corandi et al. (2010). A presença de cristais, provavelmente de oxalato de cálcio, nas células parenquimáticas mesmo após a carbonização da madeira também foi observado por Gonçalves et al. (2012) para diversas espécies de madeiras do cerrado e recentemente por Gasson et al. (2017) para Croton sonderianus sob diferentes temperaturas de carbonização.

A Tabela 2 apresenta o resultado quantitativo (média e desvio padrão) da anatomia da madeira e do carvão vegetal, assim como a comparação entre as médias obtidas para cada parâmetro anatômico avaliado antes e após a carbonização.

Tabela 2: Dados anatômicos quantitativos dos parâmetros anatômicos analisados e comparação das médias entre a madeira e o carvão da espécie Alexa grandiflora

\begin{tabular}{|c|c|c|c|c|c|c|}
\hline & & $\mathrm{DV}(\mu \mathrm{m})$ & $\mathrm{FV}\left(\mathrm{mm}^{2}\right)$ & $\mathrm{FR}(\mathrm{mm})$ & $\mathrm{AR}(\mu \mathrm{m})$ & $\mathrm{LR}(\mu \mathrm{m})$ \\
\hline Madeira & $\begin{array}{l}\text { Média } \\
\text { (Desvio Padrão) }\end{array}$ & $\begin{array}{l}270,48 \mathrm{~A} \\
(47,11)\end{array}$ & $\begin{array}{l}2,37 \mathrm{~A} \\
(1,54)\end{array}$ & $\begin{array}{l}7,03 \mathrm{~A} \\
(1,07)\end{array}$ & $\begin{array}{l}306,53 \mathrm{~B} \\
(39,09)\end{array}$ & $\begin{array}{l}40,58 \mathrm{~A} \\
(7,73)\end{array}$ \\
\hline
\end{tabular}




\begin{tabular}{|c|c|c|c|c|c|c|}
\hline Carvão & $\begin{array}{l}\text { Média } \\
\text { (Desvio Padrão) }\end{array}$ & $\begin{array}{l}187,76 \mathrm{~B} \\
(31,79)\end{array}$ & $\begin{array}{l}4,47 \mathrm{~B} \\
(1,46)\end{array}$ & $\begin{array}{l}8,13 \mathrm{~B} \\
(1,70)\end{array}$ & $\begin{array}{l}245,22 \mathrm{~A} \\
(45,64)\end{array}$ & $\begin{array}{l}24,56 \mathrm{~B} \\
(5,14)\end{array}$ \\
\hline Aument & dução \% & 30,58 & 88,61 & 15,65 & 20,00 & 39,48 \\
\hline
\end{tabular}

A análise da Tabela 2 permite observar que todos os parâmetros anatômicos quantitativos avaliados apresentaram diferenças estatisticamente significativas entre a madeira e o carvão. A quantidade de vasos por $\mathrm{mm}^{2}$ e raios por mm linear, teve aumento próximo a 88 e $15 \%$ após carbonização. Para os parâmetros mensurados, diâmetro dos vasos, altura e largura dos raios, comportamento inverso foi observado, após a carbonização houve redução significativa da dimensão dessas células, com destaque para a redução de $30 \%$ do diâmetro dos vasos e de $39 \%$ da largura dos raios após a carbonização.

A quantidade e dimensão das células demonstraram padrão opostos, após a carbonização houve aumento e redução respectivamente. A redução da dimensão celular, principalmente do diâmetro tangencial dos vasos, tem sido um padrão comumente descrito após carbonização da madeira (GONÇALVES et al., 2012; NISGOSKI et al., 2014; GASSON et al., 2017). A quantidade de parênquima axial paratraqueal pode ter influência sobre estas variações dimensionais, visto que esses grupos de células possuem parede delgada assim as tensões internas derivadas do processo de carbonização geram retrações com mais facilidade na estrutura anatômica. Gonçalves et al. (2012) relataram redução mais acentuada após carbonização para o diâmetro dos vasos das espécies que apresentaram parênquima axial paratraqueal abundante, enquanto aqueles com parênquima axial difuso tiveram pouca o nenhuma variação dimensional destas células. Quanto à alteração da quantidade de células por área, é possível que as contrações anisotrópicas do material decorrentes da carbonização tendem promover redução do volume, maior na madeira e menor carvão, o que leva a aproximação entre as células, aumentando a frequência de vasos e raios por exemplo.

O fato de não terem sido observadas alterações qualitativas entre a anatomia da madeira e do carvão indica que a manutenção da estrutura anatômica no carvão vegetal contribui para a distinção do material, auxiliando na fiscalização e conservação ambiental (GONÇALVES et al., 2016). A ausência de materiais de referência e a não constância no treinamento dos agentes responsáveis pelo controle do comércio de carvão vegetal são responsáveis pela formação ineficiente dos agentes responsáveis pelo controle do comércio de carvão vegetal, contribuindo assim para uma fiscalização inoperante. As eletromicrografia do carvão vegetal (Figura 1 - D,E,F) podem facilitar a identificação da estrutura anatômica do 
carvão mesmo em somente com lupas conta fios, Visto que o padrão e visibilidade do parênquima para espécie se mantém.

O controle efetivo do comércio de carvão vegetal pode diminuir algumas anomalias sociais derivadas da produção ilegal, como os casos de escravidão contemporânea, expondo os trabalhadores a restrições de liberdade, remuneração insatisfatória, trabalho forçado, além disso, provocando danos à saúde e bem estar dos operários, o estado do Pará é um dos estados brasileiros com maior número de trabalho análogo ao escravo (GREENPEACE, 2012).

Entretanto, a utilização de carvão vegetal proveniente de plantios ou manejo de florestas naturais é benéfica ao meio ambiente, podendo gerar saldo positivo em créditos de carbono e diminuição da emissão de efluentes no ar atmosférico, assim como viável energeticamente. Afonso et al. (2015) observaram que espécies do gênero Eucalyptus, oriundas de florestas plantadas, podem apresentar poder calorífico superiores, além de gerar menor quantidade de cinzas e voláteis durante o processo de produção se comparada com espécies nativas de Moçambique, dessa forma, a variação do método de produção de energia poderia gerar impactos ecológicos e socioeconômicos.

\section{CONCLUSÕES}

As estruturas anatômicas das espécies analisadas em sua maioria foram mantidas após o processo de carbonização, contribuindo assim para a identificação da espécie que lhe originou. Entretanto, derivado do processo de carbonização foram verificados modificações morfométricas relacionadas à dimensão e frequência dos elementos anatômicos, que devem ser consideradas no processo de identificação do carvão durante a fiscalização.

A partir do exposto, acredita-se que o presente trabalho: 1) fornece informações em literatura técnico-cientifica a respeito do comportamento de uma espécie amazônica ao processo de carbonização; 2) fornece informações para a formação técnica de agentes de fiscalização, dessa forma, auxiliando ao controle do comércio ilegal de madeira e carvão vegetal, contribuindo assim para a diminuição da perda e pressão biológica de espécies amazônicas e de impactos socioeconômicos da região.

\section{REFERÊNCIAS}

AFONSO, C.I.A.; GONÇALVES, T.A.P.; MUÑIZ, G.I.B. de; MATOS, J.L.M. de; NISGOSKI, S. Mozambique's charcoals: anatomy of nine native species. BOSQUE, v. 36(1), p. $105-112,2015$

BURGUER, L.M.; RICHTER, H.G. Anatomia da madeira. Nobel, São Paulo, 1991. 
CORANDIN, V. P. R.; CAMARGO, J. A. A.; PASTORE, T. C. M.; CRISTO, A. G. Madeiras comerciais do Brasil: chave interativa de identificação baseada em caracteres gerais e macroscópicos. Serviço Florestal Brasileiro, Laboratório de Produtos Florestais: Brasília, 2010. CR-ROM.

GONCALVES, T. A. P.; MARCATI, C. R.; SCHEEL-YBERT, R. The effect of carbonization on wood structure of Dalbergia violacea, Stryphnodendron polyphyllum, Tapirira guianensis, Vochysia tucanorum, and Pouteria torta from the brazilian cerrado. Iawa Journal, v. 33, n. 1, p. 73-90, 2012.

GONÇALVES, T. A. P.; BALlaRIN, A. W.; NISGOSKI, S.; MUÑIZ, G. I. B. A Contribution to the identification of charcoal origin in Brazil I - anatomical characterization of Corymbia and Eucalyptus. Maderas. Ciencia y tecnologia, Bío-Bío, v. 16, n. 3, p. 323336, 2014.

GONÇALVES, T. A. P.; NISGOSKI, S.; OLIVEIRA, J. S.; MARCATI, C. R.; BALLARIN, A. W.; MUÑIZ, G. I. B. A contribution to the identification of charcoal origin in Brazil II Macroscopic characterization of Cerrado species. Anais da Academia Brasileira de Ciências, v. 88, n.2, p.1045-1054, 2016.

GREENPEACE. Carvoaria Amazônia: Como a indústria de aço e ferro gusa está destruindo a floresta com a participação de governos. Manaus - AM. 17p. 2012. Disponível em <http://www.greenpeace.org/brasil/Global/brasil/documentos/2012/42\%2020P ig\%20Iron\%20D3_portugues.pdf >. Acesso em: 18 set. 2018.

HUBBEL, S.P.; HE F.; CONDIT, R.; BORDA-DE-ÁGUA, L.; KELLNER, J.; STEEGE, H. ter. How many tree species are there in the Amazon and how many of them will go extinct? PNAS, v. 105,11498-11504, 2008.

ICMBIO. Instituto Chico Mendes de Conservação da Biodiversidade. STCP Engenharia de Projetos Ltda. Plano de pesquisa geossistemas ferruginosos da Floresta Nacional de Carajás: temas prioritários.... Brasília: MMA, 2017.

INTERNATIONAL ASSOCIATION OF WOOD ANATOMISTS. List of microscope features for hardwood identification. IAWA Bulletin, Leiden, v. 10, p. 234-332, 1989.

MUÑIZ, G. I. B.; NISGOSKI, S.; FRANÇA, R. F.; SCHARDOSIN, F. Z. Anatomia do carvão de espécies florestais. Cerne, v. 18, n. 3, p. 471-477, 2012.

PEREIRA, B.L.C.; CARVALHO, A.M.M.L.; OLIVEIRA, C.O.; SANTOS, C.L.; CARNEIRO, A.C.O. de; MAGALHÃES, M.A. de. Efeito da carbonização da madeira na estrutura anatômica e densidade do carvão vegetal de Eucalyptus. Ciência Florestal, Santa Maria, v. 26, n. 2, p. 545-557, 2016

SCHEEL-YBERT, R.; KLÖKLER, D.; GASPAR, M.D.; FIGUTI, L. Proposta de amostragem padronizada para macro-vestígios bioarqueológicos: antracologia, arqueobotânica, zooarqueologia. Revista do Museu de Arqueologia e Etnologia, n. 15-16, p. 139-163, 2006.

SONTER, L. J.; HERRERA , D.; BARRETT, D. J.; GALFORD G. L.; MORAN, C. J.; 
SOARES-FILHO, B. S. Mining drives extensive deforestation in the Brazilian Amazon. Nature, v. 8, n. 1, 2017.

FEDALTO, L.C.; MENDES, I.C.A. da; CORANDIN, V.T.R. Madeiras da Amazonia. Descricao do lenho de 40 especies ocorrentes na Floresta Nacional do Tapajos. Inst. Bras. Meio Ambiente e Recurs. Nat. Renov. IBAMA: Brasilia, 156 pp. 1989

NISGOSKI, S.; MUÑIZ, G.I.B. de; BATISTA, F.R.R.; MOLLEKEN, R.E. Influence of carbonization temperature on the anatomical characteristics of Ocotea porosa (Nees \& Mart. Ex Nees) L. Barroso. Wood Sci Technol, 48:301-309. 2014 